\title{
Prevalencia del hostigamiento escolar en las instituciones públicas de Bucaramanga-Colombia*
}

\section{Prevalence of School Bullying at State Schools in Bucaramanga city, Colombia}

\author{
Yolima Ivonne Beltrán Villamizar \\ Omar Elías Torrado Duarte \\ Carlos Guillermo Vargas Beltrán
}

Grupo de investigaciones educativas Atenea

Universidad Industrial de Santander, Bogotá,

Colombia.

Cómo citar: Beltrán, YI; Torrado, O.E., Vargas, C.G. (2016) Prevalencia del Hostigamiento Escolar en las Instituciones Públicas de Bucaramanga-Colombia. Sophia 12 (2): 173-186.

\section{Resumen}

El objetivo del artículo es determinar la prevalencia del hostigamiento escolar presente en las instituciones educativas públicas de Bucaramanga-Colombia mediante una investigación transversal de corte cuantitativo y un diseño no experimental. La muestra estuvo constituida por 1776 estudiantes (48,9\% mujeres y 50,5\% hombres) pertenecientes a los grados de $4^{\circ}$ a $11^{\circ}$. El estudio se desarrolló en tres fases: caracterización de las instituciones educativas públicas de Bucaramanga; selección aleatoria de los grupos escolares sobre los que se recolectó la información; recolección y análisis de datos. Los resultados muestran una prevalencia de victimización $8,1 \%$ para el caso del hostigamiento escolar perpetrado de manera personal y del $5 \%$ para el hostigamiento escolar realizado a través de medios virtuales.

Palabras clave: Agresión, ciencias de la educación, contexto escolar, educación básica, violencia.

\begin{abstract}
The objective of this article is to determine the prevalence of bullying present in public educational institutions in Bucaramanga - Colombia through a cross- cutting quantitative research and a non- experimental design. The sample consisted of 1776 students ( $48.9 \%$ female and $50.5 \%$ male ) belonging to grades 4 to 11 th. The study was conducted in three phases: characterization of public educational institutions Bucaramanga; random selection of school groups on which the information was collected; data collection and analysis. The results show a $8.1 \%$ prevalence of victimization in the case of bullying committed personally and $5 \%$ to bullying conducted through virtual means.
\end{abstract}

Keywords: Organization change, secondary education, education policy, government policy, education sciences.

*Este estudio se desarrolló en el marco del proyecto de investigación, financiado por el Departamento Administrativo de Ciencia, Tecnología e Innovación y la Universidad Industrial de Santander, denominado: Efectividad de los Programas OBPP - Bergen y Save en la Disminución del Hostigamiento Escolar en Instituciones Públicas de Educación Básica de Bucaramanga, código 8246. 


\section{Introducción}

El hostigamiento escolar consiste en maltrato reiterado e intencionado entre iguales, mediante agresión física y/o psicológica, ejercida de un estudiante sobre otro en forma prolongada, e implica una relación interpersonal caracterizada por un desequilibrio de poder o fuerza, ya sea real o percibida (Olweus, 1993). Así, el hostigamiento escolar forma parte de la violencia escolar, toda vez que esta, comprende los actos de daño, agresión y amenaza intencionales y sistemáticos, que se manifiestan en diferentes formas y niveles, clasificados en seis categorías de comportamiento antisocial: disrupción en las aulas, problemas de disciplina (conflictos entre profesores y estudiantes), hostigamiento entre iguales (bullying), vandalismo y daños materiales, violencia física (agresiones, extorsiones, etc.) y acoso sexual (Chaux, 2012; Moreno, 2007).

En la literatura (Rodríguez, 2009) se señala que el hostigamiento escolar deriva de un tipo de relación interpersonal desarrollado en un grupo, que tiene el propósito de producir daño y humillación, y se caracteriza por conductas reiteradas de intimidación y exclusión, dirigidas a un individuo que se encuentra en posición de desventaja. El hostigamiento escolar se describe como una forma de violencia entre iguales que incluye conductas de abuso de poder, que son repetitivas y prolongadas en el tiempo, provocadas por un alumno (agresor), apoyado generalmente de un grupo (espectadores), contra una víctima indefensa que no puede salir por sí misma de esta situación; aquellas conductas se mantienen por la ignorancia $\mathrm{y}$ pasividad de las personas que no intervienen directamente en esta problemática (Díaz-Aguado, 2005).

Los principales actores involucrados en esta problemática son: el agresor (quien provoca daño o miedo a una persona); la víctima (persona receptora de las agresiones) y los espectadores o testigos, que se dividen en cuatro categorías: indiferentes (que no intervienen), culpabilizados (no intervienen por miedo al agresor, pero se sienten culpables por ello), amorales (justifican el poder que tiene el agresor sobre la víctima) y los que intervienen, ya sea animando al agresor o defendiendo a la víctima (Martín, Lobato, \& Gómez, 2007).
En lo referente a las víctimas, se han identificado dos clases: la provocadora y la pasiva o sumisa. Las primeras, muestran una combinación de ansiedad con reacción agresiva que por lo general, se percibe como desafiante, lo que el agresor suele utilizar como excusa para realizar sus actos de hostigamiento. Este tipo de respuestas defensivas inapropiadas suelen ser motivadas por la valoración positiva de la violencia en el grupo familiar, donde se incita a las víctimas a responder a las agresiones por la misma vía de la agresión (García, Guerrero \& Ortiz, 2012). Por su parte, las víctimas pasivas demuestran inseguridad, ansiedad y sumisión, y a la vez se muestran físicamente débiles, con actitud negativa ante la violencia y ante las agresiones, suelen huir o llorar. En la mayoría de las ocasiones las víctimas pasivas vivencian su experiencia negativa en silencio y permanecen solitarios en la institución educativa (Olweus, 1998).

Dentro de los factores predisponentes al hostigamiento escolar, se destaca que los estudiantes hostigados carecen de una red social consolidada, presentan falencias en la integración social y vulnerabilidad escolar; además presentan dificultades en sus relaciones sociales, lo que ocasiona bajo estatus en el grupo y su estigmatización como débiles (Avilés, 2009a). Otro factor que predispone la aparición del hostigamiento escolar, se relaciona con los rasgos físicos de las víctimas, que pueden llevar a que los agresores se interesen más por estos estudiantes que por otros; por ejemplo, se ha observado una alta tasa de hostigamiento escolar hacia estudiantes obesos, con dificultades visuales o en el habla, con discapacidades físicas, déficit cognitivo, además de elementos culturales o de género (Moreno, 2007).

Diversos estudios (Amemiya, Oliveros, \& Barrientos, 2009; Batsche, \& Knoff, 1994; Chaux, 2012; Olweus, 1998) han establecido que las principales consecuencias que experimentan los niños, niñas y adolescentes víctimas de hostigamiento escolar, están relacionadas con problemas de salud física, emocional y social. Ellas consideran a la institución educativa como un lugar inseguro y suelen rehusarse a asistir a ella; en los Estados Unidos hasta el 7\% de los estudiantes se queda en el hogar por lo menos una vez a la semana debido al hostigamiento recibido. Las víctimas reportan dificultades para conciliar 
el sueño, enuresis, dolor abdominal, cefaleas, depresión, ansiedad, baja autoestima, déficit en habilidades sociales, aislamiento social e ideación suicida. Adicionalmente se ha identificado un mayor riesgo de comportamientos autodestructivos y hetero-destructivos (Milicic, \& López, 2008).

Frente al perfil de los hostigadores, diversos estudios muestran características relacionadas con mayor fortaleza física respecto a sus víctimas y sus compañeros en general, poca preocupación por los sentimientos ajenos, pueden mostrarse depresivos, impulsivos, hostiles, demostrar poca empatía, altos niveles de autoestima, presentar déficit en habilidades comunicativas y resolución de conflictos, además de agresión y manipulación. Se describen dos categorías de hostigadores: los activos, que en forma directa agreden a su víctima; y los pasivos, que participan o apoyan la planificación del acto acosador, pero no actúan en el mismo (Olweus, 1998). Entre las posibles motivaciones del hostigador se encuentra la necesidad de poder y dominio ante sus semejantes. Las dificultades familiares juegan un papel determinante, ya que muchos de los agresores han experimentado conflictos familiares. Igualmente, se identifica como un componente causal de hostigamiento, el beneficio propio, ya que al ejercer su conducta hostigadora, un estudiante puede obtener dinero, comida, objetos de valor y reconocimiento social (Olweus, 2007).

Por su parte, los testigos o espectadores, quienes no toman un rol activo en la situación de hostigamiento y prefieren "no hacer nada", se ven igualmente intimidados por el agresor, por lo que inhiben su motivación de brindar ayuda a las víctimas. En la literatura existente al respecto se señala que el hostigamiento escolar produce una incitación en la participación en los actos de acoso, a pesar de que estos no sean los autores iníciales del evento hostil. Otro elemento que caracteriza a los espectadores es que desarrollan resistencia en relacionarse con las víctimas por temor a ser rechazadas por los demás compañeros o a convertirse en víctimas también (Defensor del Pueblo (1999; Olweus, 1998).

Los principales avances obtenidos en políticas educativas contra el hostigamiento escolar se han dado en los Países Nórdicos y el Reino Unido, donde las propuestas de Olweus, Smith y Sharp respectivamente, han sido incorporadas en las propuestas de intervención de los ministerios de educación. En el caso del Reino Unido, ha sido implementado un Código de Conducta Específico sobre hostigamiento escolar entre iguales, de uso obligatorio para las instituciones educativas (Defensor del Pueblo, 1999). Asimismo, Noruega, Finlandia y Suecia principalmente, desarrollan estrategias que involucran no solo a las escuelas sino a toda la sociedad, estableciendo leyes estatales en tres ámbitos diferentes: política nacional, política comunitaria y política escolar nacional (Defensor del Pueblo, 1999). Desde 1998 existe el Observatorio Europeo de la Violencia Escolar, cuya finalidad es consolidar una amplia base de datos internacionales de fácil acceso y a su vez, ampliar sus investigaciones en temas próximos a problemas de violencia social, con el fin de crear conceptos claros y comunes sobre hostigamiento y violencia escolar, tanto para la comunidad investigadora, como la escolar (Ortega-Ruíz, 1998).

En América Latina, países como México, Argentina, Costa Rica, Nicaragua, Chile y Perú, entre otros, han realizado estudios descriptivos de este fenómeno $\mathrm{y}$ han implementado programas de atención $\mathrm{y}$ prevención del hostigamiento escolar. Sin embargo, no se evidencian políticas educativas concretas, encaminadas a su prevención, disminución y atención. No obstante, los ministerios de educación de los respectivos países han propuesto programas y estrategias orientadas a la erradicación de la violencia, al fomento del buen trato, la prevención del maltrato y la formación escolar en convivencia, que sin embargo no han sido evaluados (Aguirre, 2009; Milicic, \& López, 2008).

En Colombia, el Congreso de la República estableció la Ley 1620 de 2013, por la cual se crea el Sistema Nacional de Convivencia Escolar y Formación para el Ejercicio de los Derechos Humanos, Sexuales y Reproductivos y la Prevención y Mitigación de la Violencia Escolar, donde se reconoce a los niños, niñas y adolescentes como sujetos de derechos y a la comunidad educativa en los niveles de preescolar, básica y media, como responsable de formar para el ejercicio de los mismos; ello, conforme a lo dispuesto en la Constitución Política Nacional, las leyes 115 de 1994 y 1098 de 2006, las disposiciones del 
Consejo Nacional de Política Social y demás normas asociadas a violencia escolar, que plantean demandas específicas al sistema escolar (Ley 1620 del 2013).

El objeto de la Ley 1620 de 2013 es contribuir a la formación de ciudadanos activos que aporten a la construcción de una sociedad democrática, participativa, pluralista e intelectual, en concordancia con el mandato constitucional y la Ley General de Educación, promoviendo y fortaleciendo la formación ciudadana y el ejercicio de los Derechos Humanos, Sexuales y Reproductivos de los estudiantes, de los niveles educativos de preescolar, básica y media (Ley 1620 del 2013). Asimismo, esta ley busca fomentar, fortalecer y articular acciones de diferentes instancias del Estado para la convivencia escolar, la construcción de ciudadanía y la educación encaminada al ejercicio de los Derechos Humanos, Sexuales y Reproductivos de los niños, niñas y adolescentes de los niveles educativos de preescolar, primaria, básica y media (Ley 1620 del 2013).

Para ello, se propone la conformación del Comité Nacional de Convivencia Escolar, integrado de manera permanente por el Ministerio de Educación Nacional (MEN) o el Viceministerio de Educación Preescolar, Básica y Media, quien lo presidirá; el Ministerio de Salud o un delegado; el Instituto Colombiano de Bienestar Familiar (ICBF) o un delegado; además de representantes no permanentes como el Alto Consejero Presidencial para la Convivencia y Seguridad Ciudadana o su delegado; el Alto Consejero Presidencial para la Equidad de la Mujer o su delegado; el Ministro de Cultura o un Viceministro delegado; el Ministro de Tecnologías de la Información y las Comunicaciones o un Viceministro delegado; el Ministro del Interior o un Viceministro delegado; el Ministro de Justicia y del Derecho o un Viceministro delegado; y el Director de la Policía de Infancia y Adolescencia o un Comandante delegado (Ley 1620 del 2013).

Dicho comité debe definir y coordinar la gestión y operación del sistema a nivel nacional, territorial y escolar, encaminando sus acciones al cumplimiento del objetivo principal de la Ley 1620 del 2013, armonizándolas con las políticas nacionales y sectoriales, así como con las estrategias y programas relacionados con la prevención y mitigación de la violencia escolar (Ley 1620 del 2013).
Asimismo, la Ley 1620 implica la conformación de comités municipales, distritales o departamentales, conformados por representantes de las secretarías de Gobierno, Educación, Cultura, el Director Regional del ICBF, las Comisarías de Familia y la Policía de Infancia y Adolescencia, así como un representante de los rectores de los establecimientos educativos; lo cuales deben garantizar la implementación de la Ruta de Atención Integral para la Convivencia Escolar en la jurisdicción respectiva y el acatamiento por todas las entidades que conforman el sistema (Gaviria, et al., 2012).

En el ámbito de cada institución educativa se ordena el establecimiento de un Comité Escolar de Convivencia, que apoyará la promoción y seguimiento a la convivencia escolar, así como al desarrollo de los manuales de convivencia, programas de prevención y mitigación de la violencia escolar; dando tratamiento y apoyo al manejo y resolución de los conflictos escolares por medio de la aplicación de los manuales de convivencia, garantizando el cumplimiento de las disposiciones establecidas en el mismo, teniendo en cuenta el conducto regular que cada institución establezca (Gaviria, et al., 2012). Dichos comités de convivencia se encuentran liderados por docentes de la institución. En congruencia, diversos autores (Chagas, 2005; García \& Ortíz, 2012) coinciden en la importancia de incluir a los maestros en la toma de decisiones y la intervención para el mejoramiento de la convivencia escolar, sin embargo, también coinciden en la necesidad de brindarles herramientas que disminuyan los errores metodológicos en los que suelen incurrir los maestros debido a juicios subjetivos y estrategias empíricas que pueden entorpecer el proceso de resolución de conflictos en el contexto escolar.

En la literatura se reportan los programas Olweus Bullying Prevention Program (OBPP) de la Universidad de Bergen, Noruega (Olweus, 1998) y el Programa Sevilla Anti-Violencia Escolar (Save) de España (Ortega, \& Del Rey, 2001). El Programa OBPP - Universidad de Bergen, utiliza un enfoque que implica medidas de intervención a nivel institucional, de aula de clase e individual; este cuenta con un cuestionario para establecer la magnitud del problema, plantea jornadas de reflexión $\mathrm{y}$ actividades de vigilancia durante el recreo y la formación de equipos de profesores involucrando 
activamente a los padres de familia (Olweus, 1998). Por su parte, el Programa Save, plantea dos líneas de intervención: la preventiva y la remedial. En el plano preventivo propone trabajar en los campos de educación en emociones, sentimientos y valores, el trabajo en grupo cooperativo y la gestión democrática de la convivencia; en la línea remedial trabaja en la resolución de conflictos, la ayuda entre iguales, los círculos de calidad, los programas de desarrollo de la empatía y los programas de desarrollo de la asertividad (Ortega, \& Del Rey, 2001).

Desde las primeras publicaciones realizadas por el psicólogo noruego Dan Olweus en la década de 1970, el hostigamiento escolar ha venido siendo un tema que acrecienta el interés de la comunidad científica con el pasar de las décadas en diversos países, siendo la prevalencia uno de los temas sobre los que se ha investigado en mayor medida (Milicic \& López, 2008; Pérez-Fuentes, Gázquez, Fernández-Baena \& Molero, 2011).

En Europa donde surgieron los primeros estudios sobre el hostigamiento escolar, se registra que al menos el $15 \%$ de los alumnos europeos han sido intimidados o agredidos alguna vez, o han presenciado alguna situación de conflicto (Debardieux, \& Blaya, 2001); revisando en detalle la prevalencia del hostigamiento escolar por naciones, se identifica que el $18,2 \%$ de los estudiantes se ha visto involucrado en episodios de hostigamiento en Noruega y Suecia, siendo el 10,1\% víctimas, el 6,5\% agresores y el 1,6\% espectadores (Solberg, \& Olweus, 2003). Para el caso de España, un estudio realizado con una muestra representativa de instituciones educativas de secundaria de la ciudad de Valladolid, muestra que el $8,1 \%$ de los alumnos se ha visto involucrado en situaciones de hostigamiento escolar, de los cuales el 5,7\% son las víctimas y el restante 5,9\% (Wang, Iannotti \& Nansel, 2009).

Por otra parte, se ha identificado (Whitney, \& Smith, 1993) en una muestra representativa de 6.000 estudiantes de Inglaterra, que el $21 \%$ se ha visto involucrado en el fenómeno del hostigamiento escolar, siendo el $14 \%$ víctimas y el $7 \%$ agresores; asimismo en Holanda, se ha identificado una prevalencia general de estudiantes involucrados en situaciones de hostigamiento escolar del 34\%, siendo el $13 \%$ víctimas, el $17 \%$ agresores y el $4 \%$ espectadores (Whitney \& Smith, 1993).
Para el caso de los Estados Unidos, utilizando una muestra compuesta por 7.182 estudiantes de secundaria de diversas escuelas alrededor de la nación, se encontró que el 20,8\% habían sido hostigados durante los dos meses anteriores a la realización del estudio, especificando que el $53.6 \%$ de estos recibió agresiones verbales, el $13.6 \%$ a través de medios virtuales y el $51.4 \%$ habría sufrido aislamiento social intencionado (Avilés, \& Monjas, 2005). El 13.3\% de los estudiantes reportó haber sido hostigadores en los últimos dos meses previos a la recolección de información, empleando la agresión verbal $(37,4 \%)$, el aislamiento social $(27,2 \%)$ y utilizando medios virtuales $(8,3 \%)$ para realizar el hostigamiento (Avilés \& Monjas, 2005).

En América Latina también se han realizado diversos estudios que permiten identificar la prevalencia del hostigamiento escolar en varios países de la región; en México, ha sido reportado que entre el $20 \%$ y $32 \%$ de los estudiantes afirma haber sido agredido en el ámbito escolar, siendo la agresión verbal la de mayor prevalencia (72\%) (Instituto Nacional de Salud Pública, 2006). En Nicaragua un estudio hecho con una muestra representativa de la ciudad de Managua, reportó una prevalencia del hostigamiento escolar de $51,7 \%$, siendo la agresión verbal el hallazgo mas recurrente y coincidente con los datos encontrados en México (Romera, Del Rey \& Ortega, 2011).

En Brasil en un estudio hecho con una muestra representativa de la ciudad de Pelotas en el estado de Rio Grande do Sul, se identificó que el $17.6 \%$ de los estudiantes había sufrido hostigamiento escolar manifestando diversos tipos de agresiones: verbales $(75,1 \%)$, físicas $(62,4 \%)$, emocionales $(23,8 \%)$, raciales $(6,3 \%)$ y sexuales $(1,1 \%)$ (Moura, Cruz, \& Quevedo, 2011) (Recha, R., Halpernb, Tedescoc, \& Santosd, 2013). También en el estado de Rio Grande do Sul, fue identificada en una muestra representativa de la ciudad de Caxias do Sul una prevalencia del $10,2 \%$ en cuanto a estudiantes agredidos, siendo en este caso la agresión física, la más reportada $(38,7 \%)$ (Recha, Halpernb, Tedescoc \& Santosd, 2013).

En Perú según estudios realizados en escolares de cuatro poblaciones del país (Ayacucho, Cusco, Junín y Lima Este), existe una prevalencia de victimización del hostigamiento escolar del 47\%, resaltándose 
la agresión física como la principal clase de intimidación $(34,8 \%)$, seguida de la agresión verbal $(34,5 \%)$, el maltrato psicológico $(9,5 \%)$ y la agresión sexual $(4,7 \%)$ (Amemiya, Oliveros, \& Barrientos, 2009; Oliveros, Figueroa, Mayorga, Cano, Quispe, \& Barrientos, 2008).

Finalmente, en el contexto colombiano en un estudio que tuvo como uno de sus objetivos, determinar el nivel de acoso escolar en los colegios de la localidad de Ciudad Bolívar de Bogotá, y en el que se aplicó un formato de encuesta a una muestra de 3.226 alumnos de educación básica y media, se identificó que el $21,8 \%$ de los alumnos ha sido víctima de hostigamiento escolar (Cepeda-Cuervo, Pacheco-Durán, García-Barco, \& Piraquive-Peña, 2008). En un estudio hecho con 14 colegios (10 privados y 4 públicos) de Cali (Colombia), de los que se seleccionó una muestra no probabilística de 2.562 estudiantes ( 1.049 hombres y 1.513 mujeres) de los grados $6^{\circ}, 7^{\circ}$ y $8^{\circ}$, se encontró que el $24.7 \%$ de los alumnos, ha sido víctima de comportamientos de intimidación como agresión verbal, física y psicológica (Paredes, Álvarez, Lega, \& Vernon, 2008).

En el caso específico del departamento de Santander, en el municipio de Floridablanca (área metropolitana de Bucaramanga) se realizó un estudio descriptivo, con una muestra de 304 estudiantes de los niveles académicos $6^{\circ}, 9^{\circ}$ y $11^{\circ}$, a través del cual se identificó una prevalencia del hostigamiento escolar del $24,7 \%$, destacando la importancia de las redes de apoyo social en la mitigación de la afectación individual, institucional y social que produce el fenómeno del hostigamiento escolar (Uribe, Orcasita, \& Aguillon, 2012).

La calidad de los programas de prevención y el control del hostigamiento escolar están sujetos al nivel de conocimiento que se tenga sobre dicho fenómeno (Wang, Iannotti, \& Nansel, 2009). Este supuesto da origen al presente trabajo, cuyo objetivo es determinar la prevalencia del hostigamiento escolar que se presenta en las instituciones educativas públicas del municipio de Bucaramanga-Colombia, para aportar conocimiento sobre el tema a la comunidad científica y a la sociedad colombiana.

\section{Metodología}

El tipo de investigación realizada es transversal descriptiva, con enfoque cuantitativo utilizando un diseño no experimental.

\section{Participantes}

Todos los involucrados en esta investigación lo hicieron de manera voluntaria con conocimiento pleno de los objetivos del estudio; por ello, se firmaron los consentimientos informados (instituciones educativas), los permisos (padres de familia) y los asentimientos (menores de edad). La población $(\mathrm{N})$ de estudiantes de las instituciones educativas públicas de la ciudad de Bucaramanga-Colombia, está conformada por 48.242 sujetos. La muestra (n) estuvo constituida por 1776 estudiantes de los grados $4^{\circ}$ a $11^{\circ}$, de ocho instituciones educativas de la ciudad, con edad promedio de 13,37 años ( $\mathrm{DE}=2,83)$. La muestra fue obtenida mediante muestreo aleatorio simple, considerando una confiabilidad de $99 \%$ y un error muestral del 3\%. La tabla 1 muestra la información demográfica de los estudiantes participantes.

Tabla 1. Información demográfica de los estudiantes participantes en el estudio

\begin{tabular}{|c|c|c|}
\hline Variable & Frecuencia & Porcentaje $(\%)$ \\
\hline \multicolumn{3}{|l|}{ Sexo } \\
\hline Mujer & 851 & 47,92 \\
\hline Hombre & 790 & 44,48 \\
\hline $\mathrm{Ns} / \mathrm{Nr}^{*}$ & 135 & 7,6 \\
\hline \multicolumn{3}{|l|}{ Edad } \\
\hline 8-10 años & 238 & 13,4 \\
\hline 11-14 años & 865 & 48,7 \\
\hline 15-17 años & 587 & 33,05 \\
\hline $18->18$ años & 50 & 2,82 \\
\hline $\mathrm{Ns} / \mathrm{Nr}^{*}$ & 36 & 2,03 \\
\hline \multicolumn{3}{|l|}{ Grado Escolar } \\
\hline $4^{\circ}$ & 179 & 10,08 \\
\hline $5^{\circ}$ & 199 & 11,2 \\
\hline $6^{\circ}$ & 273 & 15,37 \\
\hline $7^{\circ}$ & 266 & 14,98 \\
\hline $8^{\circ}$ & 186 & 10,47 \\
\hline $9^{\circ}$ & 212 & 11,94 \\
\hline $10^{\circ}$ & 257 & 14,47 \\
\hline $11^{\circ}$ & 204 & 11,49 \\
\hline
\end{tabular}

*No sabe / No responde Fuente: elaboración propia 
Como criterio de inclusión, se estableció que el/ la estudiante contara con el permiso escrito de su padre/madre o acudiente, firmara el formato de asentimiento informado para la participación en el estudio, estuviese matriculado(a) en la institución seleccionada y que perteneciera al grupo escolar elegido para la recolección de la información.

\section{Instrumentos}

Inicialmente, para el desarrollo del estudio se emplearon tres formatos, dos de consentimiento informado (uno institucional y otro para padres/ madres) y un formato de asentimiento informado (para menores de edad), los cuales presentan al lector la justificación y los objetivos de la investigación, los procedimientos a emplear, las molestias o los riesgos esperados, los beneficios potenciales, la garantía de recibir respuesta a cualquier pregunta y aclaración a cualquier duda acerca de los procedimientos, riesgos, beneficios y otros asuntos relacionados con la investigación, la libertad de retirar su consentimiento en cualquier momento y dejar de participar en el estudio, la seguridad y confidencialidad de la información, y demás aspectos que se establecen en el artículo 15 de la Resolución № 008430 de 1993.

En el proceso de recolección de información, primero se empleó el Formato de Caracterización Institucional, que consta de quince ítems encaminados a indagar sobre la instituciones educativas, datos de ubicación y contacto, marco estratégico, infraestructura, número de sedes y de estudiantes en relación con los grados académicos, sexo y jornada, el número de profesores por sede y su formación; por último, el formato indaga sobre los proyectos institucionales o alianzas que tenga el plantel y que se estén ejecutando al momento de la visita.

Seguidamente se presentó a los estudiantes, el "Cuestionario sobre el estado inicial de la convivencia escolar, Forma A, Estudiantes", conformado por noventa y un (91) ítems, divididas en tres partes. La primera parte, consta de quince (15) preguntas, donde se solicitan datos de identificación de los estudiantes como el grado escolar, la edad, la conformación familiar y la autopercepción física. La parte dos, con veintisiete (27) ítems, indaga las dinámicas familiares de los estudiantes, las posibles dificultades en el hogar y las causas de conflicto. La parte tres constituida por cuarenta y nueve (49) preguntas, busca identificar las situaciones de rechazo o maltrato físico o psicológico que hayan recibido en la institución. Las catorce (14) preguntas iníciales de la parte tres consultan el tipo de maltrato que se da, la frecuencia del mismo, las respuestas comportamentales ante las agresiones hacia él o hacia un tercero; seguidamente se indaga, por medio de siete (7) preguntas, las posibles discriminaciones ejercidas, observadas o recibidas por el color de piel, la religión, cultura o procedencia extranjera; posteriormente veintiocho (28) preguntas se enfocan en examinar las posibles agresiones ejercidas o recibidas por los estudiantes a través del celular y/o internet, la frecuencia de los maltratos, las plataformas por las cuales se dan y la respuesta ante el maltrato por estos medios hacia él o un tercero.

\section{Procedimiento}

Para el desarrollo de la investigación se ejecutaron tres fases metodológicas; la primera, fue la identificación de cada una de las instituciones educativas de carácter público en la ciudad de Bucaramanga-Colombia, para su posterior caracterización, según el Formato de Caracterización Institucional; en esta etapa, los directivos realizaron la firma del formato de consentimiento informado institucional.

Una vez identificadas las instituciones y conociendo los detalles de su funcionamiento administrativo y número de estudiantes, se procedió a la segunda fase, donde se seleccionaron de forma aleatoria, los grupos escolares sobre los que se recolectaría la información requerida en cada institución educativa. En esta fase, se procedió a la socialización de la propuesta de investigación, realizando jornadas grupales con la comunidad educativa (directivos, docentes, padres de familia y estudiantes); asimismo, se solicitó a los padres de familia el respectivo permiso firmado sobre la participación de sus hijos y a los estudiantes su asentimiento informado por medio escrito.

Finalmente, la tercera fase estuvo dirigida a la recolección de información, mediante el "Cuestionario sobre el estado inicial de la convivencia escolar, Forma A, Estudiantes", para la posterior tabulación y análisis de datos, terminando en la sistematización de los informes de resultados que serían socializados en las instituciones participantes. 


\section{Consideraciones éticas}

La presente se establece como una investigación de riesgo mínimo según lo establecido en el artículo 6 de la resolución 8430 de 1993, donde se indican los criterios técnicos y administrativos de la investigación con seres humanos en Colombia. Teniendo en cuenta que esta investigación implica intervención con niños, niñas y adolescentes, se consideraron las disposiciones legales vigentes sobre los aspectos éticos a tener en cuenta con dicha población, como el Código de Infancia y Adolescencia (Ley 1098), la Resolución 8430 de 1993 (Procedimientos de Consentimiento Informado y Asentimiento Informado) y la Ley 1090 de 2006 (Código Deontológico y Bioético del ejercicio de la Psicología en Colombia).

Esta investigación se realizó con la expresa autorización de la Universidad Industrial de Santander (UIS), de los directivos de las instituciones de educación básica y media participantes y del Comité de Ética designado por la UIS que avaló la propuesta.

\section{Resultados}

A través de un análisis de frecuencias se logró identificar una prevalencia del $8,1 \%$ de estudiantes que reportan ser víctimas de intimidación, rechazo o maltrato físico o psicológico por algunos compañeros, al menos una vez a la semana, durante los tres meses previos a la recolección de información. El 54,8\% de los estudiantes agredidos son mujeres y el restante $45,2 \%$ hombres. En relación con el hostigamiento escolar perpetrado a través de medios virtuales, se identificó una prevalencia del $5 \%$, siendo en este caso más afectados los hombres (57,9\%) que las mujeres $(42,1 \%)$.

Haciendo la distinción por niveles escolares, se observa que en el grado $11^{\circ}$ no hay ningún reporte de estudiantes víctimas de hostigamiento escolar, mientras que en $8^{\circ}$ presenta el pico superior de casos reportados, representados en el $25,8 \%$ de las víctimas identificadas. Para el fenómeno del hostigamiento escolar realizado por medios virtuales, los grados $6^{\circ}$, $7^{\circ}$ y $9^{\circ}$ son los de menor número de víctimas (un caso cada uno); de igual manera, en las agresiones a través de medios virtuales el grado $8^{\circ}$ es el que presenta el mayor número de víctimas identificadas, $26,3 \%$.
Adicionalmente, se halló que las víctimas refieren en mayor medida $(58,1 \%$ agresión personal y $26,3 \%$ a través de medios virtuales) que estas son perpetradas por grupos de estudiantes, aunque se presenta una distinción por géneros según el tipo de hostigamiento, pues para el caso del escolar personal, se referencia principalmente a los grupos de niños $(38,7 \%)$ y para el caso del hostigamiento escolar ejercido mediante medios virtuales, se reportan los grupos de niños y niñas $(26,3 \%)$. La tabla 2 presenta en detalle la información encontrada sobre la prevalencia de hostigamiento escolar por género, por grados y por la tipología de los agresores.

Tabla 2. Frecuencia y porcentaje del hostigamiento escolar (personal y virtual) por género, grado escolar y tipología del agresor(es).

\begin{tabular}{lcc}
\hline & $\begin{array}{c}\text { Hostigamiento Escolar físico } \\
\text { o psicológico }\end{array}$ & $\begin{array}{c}\text { Hostigamiento Escolar por } \\
\text { medios virtuales }\end{array}$ \\
\cline { 2 - 3 } \multicolumn{1}{c}{ Categoría } & Porcentaje & Porcentaje \\
\hline Total & 8,1 & 5 \\
Sexo & & \\
Mujeres & 54,8 & 42,1 \\
Hombres & 45,2 & 57,9 \\
\hline Grado Escolar & & \\
$4^{\circ}$ & 12,9 & 15,8 \\
$5^{\circ}$ & 22,6 & 15,8 \\
$6^{\circ}$ & 9,7 & 5,3 \\
$7^{\circ}$ & 12,9 & 5,3 \\
$8^{\circ}$ & 25,8 & 26,3 \\
$9^{\circ}$ & 3,2 & 5,3 \\
$10^{\circ}$ & 12,9 & 15,8 \\
$11^{\circ}$ & 0 & 10,5 \\
\hline Tipo de Agresor & & \\
Una niña & 3,2 & 0 \\
Un niño & 19,4 & 10,5 \\
Un grupo de niñas & 6,5 & 2,6 \\
Un grupo de niños & 38,7 & 47,4 \\
Un grupo de niños y niñas & 12,9 & \\
Ns / Nr* & & \\
\hline & & \\
\hline
\end{tabular}

*No sabe / No responde Fuente: elaboración propia

$\mathrm{Al}$ indagar sobre las situaciones de agresión, mediante la presentación a los estudiantes de opciones de respuesta con posibilidad de múltiple respuesta sobre los tipos de violencia que ocurren en las instituciones educativas públicas de Bucaramanga, se encontró que el principal tipo de agresión es la verbal (palabras 
hirientes) que representan el 61,3\% de los estudiantes identificados como víctimas del hostigamiento escolar en forma personal; seguidamente están los sobrenombres $(58,1 \%)$, entendidos como calificativos negativos realizados hacia un estudiante, y las calumnias o acusaciones falsas que se levantan en contra de un individuo $(54,8 \%)$. A su vez, más de la mitad de los estudiantes $(58,1 \%)$ que reportan ser víctimas de hostigamiento escolar, refieren que estas situaciones se presentan en los salones de clase, seguido de escenarios callejeros alrededor del colegio $(45,2 \%)$ y el patio escolar $(25,8 \%)$.

La tabla 3 presenta datos sobre las principales conductas de respuesta ante las situaciones de agresión, que emiten los estudiantes víctimas, identificándose que en su mayoría, éstos tienden a ignorar los episodios que a menudo se les presentan $(83,9 \%)$; a la vez que se observa que los docentes se convierten en el principal apoyo para los estudiantes, pues son a quienes más se les comunica sobre este tipo de situaciones $(35,5 \%)$, y quienes mayor intervención realizan en ellas $(41,9 \%)$.

Tabla 3. Frecuencia y porcentaje de respuestas emitidas por las víctimas del hostigamiento escolar, sobre el contexto en que se presentan las agresiones

\begin{tabular}{lc}
\hline \multicolumn{1}{c}{ Categoría } & $\begin{array}{c}\text { Porcentaje de estudiantes } \\
\text { afectados }\end{array}$ \\
\hline Tipo de agresión & 38,7 \\
Agresión Física & 22,6 \\
Robos & 61,3 \\
Agresión verbal & 58,1 \\
Sobrenombres & 54,8 \\
Calumnias & 12,9 \\
Amenazas & 9,7 \\
Rechazo social & 12,9 \\
Daño de artículos personales & \\
\hline Lugar de la Agresión & 58,1 \\
Salón de clases & 25,8 \\
Patio escolar & 16,1 \\
Cancha escolar & 45,2 \\
En la calle & 6,5 \\
Baños escolares & 6,5 \\
Cafetería & \\
\hline Respuesta ante la agresión & 83,9 \\
\hline Ignorar la situación & 38,7 \\
Llorar & 35,5 \\
Hablar con el agresor para que deje de hacerlo & 22,6 \\
\hline He intentado hacer lo mismo al agresor & \\
\hline Personas a quien comunica la situación & 25,8 \\
\hline Amigos & 22,6 \\
Padres & 35,5 \\
Docentes & 16,1 \\
\hline Persona que interviene & 29 \\
\hline Nadie & 19,4 \\
Amigos & 41,9 \\
\hline Padres & \\
Docentes & \\
\hline & \\
& \\
\hline
\end{tabular}

Entre tanto, se estableció que el 6,5\% de los estudiantes encuestados indicaron haber agredido física o psicológicamente a alguno de sus compañeros de institución, al menos una vez por semana.

Por otra parte, en relación con el hostigamiento escolar ejercido a través de medios virtuales, se halló que del 5\% de la muestra reporta ser víctima de hostigamiento escolar por medios virtuales, el 36,8\% refiere haber sido agredido por medio de su teléfono móvil, el 31,6\% a través de internet y quienes son agredidos por ambos medios (teléfono móvil e internet) constituyen el restante $31,6 \%$.

Los estudiantes víctimas de hostigamiento escolar a través de medios virtuales reportan que su principal respuesta ante la agresión es el llanto $(57,9 \%)$, mientras que los que son agredidos personalmente, reportan que ignoran la situación (83,9\%); asimismo, se observa una diferencia respecto a las personas a quienes se comunica la situación, pues mientras los estudiantes agredidos personalmente refieren que comunican la situación a sus docentes $(41,9 \%)$, las víctimas de hostigamiento virtual lo hacen a sus docentes $(68,4 \%)$.

Tabla 4. Frecuencia y porcentaje del medio utilizado en el hostigamiento virtual, y respuestas de los agredidos.

\begin{tabular}{ll}
\hline \multicolumn{1}{c}{ Categoría } & $\begin{array}{c}\text { Porcentaje de } \\
\text { estudiantes afectados }\end{array}$ \\
\hline Medio empleado para la agresión & 36,8 \\
Teléfono móvil & 31,6 \\
Internet & 31,6 \\
Ambos & \\
\hline Respuesta ante la agresión & 26,3 \\
\hline Ignorar la situación & 57,9 \\
Llorar & 10,5 \\
Hablar con el agresor para que deje de hacerlo & 10,5 \\
He intentado hacer lo mismo al agresor & \\
\hline Personas a quien comunica la situación & 68,4 \\
\hline Amigos & 10,5 \\
Padres & 10,5 \\
Docentes & 10,5 \\
Ns / Nr*
\end{tabular}

*No sabe / No responde

Fuente: elaboración propia 
Con respecto a los estudiantes que refieren haber agredido a uno de sus pares al menos una vez por semana empleando el teléfono móvil, internet o ambos, se obtuvo una prevalencia del 2,1\%. Asimismo, se encontró que del total de la muestra de estudiantes, el 9,2\% expresan que en algún momento del año lectivo, en que se realizó la recolección de información (año 2013), han sentido miedo de asistir a la institución educativa a la que pertenecen debido al hostigamiento escolar que se presenta en ella.

\section{Discusión de resultados}

En el presente estudio se analizó la prevalencia del fenómeno del hostigamiento escolar que se da en forma personal o de manera virtual, en los estudiantes de las instituciones educativas públicas de la ciudad de Bucaramanga-Colombia, considerándose la ocurrencia diferencial entre géneros y grados escolares, e identificando el contexto en que se presentan las situaciones de agresión.

El hostigamiento escolar ha sido considerado un fenómeno universal (Oliveros, Figueroa, Mayorga, Cano, Quispe, \& Barrientos, 2008) debido a su prevalencia en diversos países del mundo en que se ha estudiado; sin embargo, numerosos estudios indican diferencias cuantitativas y cualitativas del fenómeno, entre latitudes (Oliveros, \& Barrientos, 2007; Oliveros, Figueroa, Mayorga, Cano, Quispe, \& Barrientos, 2008). El estudio del hostigamiento escolar cobra importancia debido a las consecuencias negativas y prolongadas en el tiempo que acarrea su presencia en una institución educativa, tanto para los estudiantes agredidos como agresores (Oliveros, Figueroa, Mayorga, Cano, Quispe, \& Barrientos, 2008; Kamen, et al., 2012).

Las instituciones educativas como entes socializadores requieren del conocimiento formal ajustado a su contexto sobre el hostigamiento escolar y necesitan generar herramientas normativas y pedagógicas que les permitan actuar frente al fenómeno y prevenirlo de manera eficaz (Kamen, et al., 2012), además de potenciar el involucramiento familiar, pues se ha reportado que el $25 \%$ de los padres/madres que tienen conocimiento de las agresiones físicas o verbales realizadas en contra de su hijo(a), restan importancia a las medidas de protección requeridas y en consecuencia no actúan (Serrano \& Iborra, 2005).
Los resultados del presente estudio, sugieren que el $8,1 \%$ de los estudiantes de las instituciones educativas públicas de Bucaramanga, fueron intimidados, rechazados o maltratados física o psicológicamente por alguno(s) de sus compañeros(as) al menos una vez a la semana, durante los tres meses previos a la recolección de información, siendo las mujeres las que representan la mayor proporción de afectados con el $54,8 \%$ y con una diferencia de 9,6 puntos porcentuales por encima de los hombres, quienes representan el 45,2\%. Esta prevalencia desciende al $5 \%$ cuando se tienen en cuenta los estudiantes que han sido intimidados, rechazados o maltratados a través de internet o el teléfono móvil sin que haya una diferencia notoria entre uno $\mathrm{u}$ otro medio utilizado para agredir (teléfono móvil $=36,8 \%$; internet $=31,6 \%$; ambos $=31,6 \%$ ), hallazgo congruente con estudios similares (Avilés, 2009a; Buelga, Cava, $\&$ Musitu, 2010). En este caso, los hombres fueron los que presentaron la mayor proporción entre los agredidos (57,9\%), estando 15,8 puntos porcentuales por encima de las mujeres agredidas $(42,1 \%)$ siendo congruente con resultados mostrados en la literatura (Avilés, 2009b).

Las diferencias metodológicas utilizadas por los diversos estudios en distintos países, complejiza la comparación de la prevalencia del hostigamiento escolar identificada en el presente estudio, debido por ejemplo, a la duración considerada en cada investigación para determinar su prevalencia y los instrumentos empleados en la recolección de información; aun así, en las instituciones educativas estudiadas en Bucaramanga se evidencia una prevalencia menor que en países como Perú, México y Brasil (Instituto Nacional de Salud Pública, 2006; Moura, Cruz \& Quevedo, 2011; Oliveros, Figueroa, Mayorga, Cano, Quispe \& Barrientos, 2008), al igual que en estudios realizados en Colombia (Paredes, Álvarez, Lega \& Vernon, 2008; Uribe, Orcasita \& Aguillon, 2012), aunque es mayor que los datos hallados en España (Wang, Iannotti \& Nansel, 2009).

Adicionalmente, es posible observar que los resultados aquí hallados respecto a la afectación por géneros, coinciden con los evidenciados en la literatura, donde se refiere que las agresiones que se dan de manera personal, son más comunes en las mujeres que en los hombres (Avilés, 2009b; 
Buelga, Cava, \& Musitu, 2010). En contraste, son contrarios a los datos de estudios que refieren que las agresiones son ocasionadas principalmente por un solo individuo y no por grupos, como se observó en esta investigación (Oliveros, Figueroa, Mayorga, Cano, Quispe \& Barrientos, 2008; Paredes, Álvarez, Lega \& Vernon, 2008).

En relación con el tipo de agresión, lo evidenciado en el presente estudio es congruente con los hallazgos de estudios similares donde se reporta que la agresión verbal $(61,3 \%)$ y los sobrenombres $(58,1 \%)$ son los tipos de agresión más reportados por las víctimas (Moura, Cruz \& Quevedo, 2011; Oliveros, Figueroa, Mayorga, Cano, Quispe \& Barrientos, 2008; Paredes, Álvarez, Lega \& Vernon, 2008) y donde se señala que el salón de clases es el lugar de más frecuente ocurrencia $(58,1 \%)$ de las situaciones de agresión (Paredes, Álvarez, Lega \& Vernon, 2008).

Adicionalmente, se encontró que $83,9 \%$ de víctimas de agresión personal y $26,3 \%$ por medios virtuales ignoran la situación de ataque a la que se enfrentan; el $38,7 \%$ por agresión personal y el $57,9 \%$ por medios virtuales reaccionan entrando en llanto, lo que es coherente con hallazgos de estudios reportados en la literatura (Moreno, 2007). El 68,4\% de las víctimas de agresión por medios virtuales informan lo que sucede a sus amigos; en el caso de las agresiones personales, el $35,5 \%$ de las víctimas reporta la situación al docente, posiblemente porque este tipo de intimidación ocurre con mayor frecuencia en el aula de clase, donde el maestro como figura de autoridad tiene la posibilidad de intervenir, tal como lo señala el $41,9 \%$ de los estudiantes víctimas de hostigamiento escolar.

Asimismo, se observan diferencias en las posibilidades de apoyo que percibe el estudiante víctima de hostigamiento escolar, según sea el modo en que se den las situaciones de agresión (personalmente o por medios virtuales), aunque se requiere ahondar en el estudio de estas características diferenciales, para determinar detalles relevantes frente al tema.

En cuanto a la diferencia por cursos de la prevalencia del hostigamiento, el presente estudio indica que en el grado $8^{\circ}$ se presenta un alto número de estudiantes víctimas de intimidación, rechazo o maltrato infringido de manera personal $(25,8 \%)$ o a través de medios virtuales $(26,3 \%)$ : en el hostigamiento escolar personal, se observa un ascenso en el número de víctimas desde el grado $6^{\circ}$ a $8^{\circ}$ (sexto $=9,7 \%$; séptimo $=12,9 \%$; octavo $=28,8$ ), siendo este último el pico más alto. Además, se observa un alto número de víctimas en el grado $5^{\circ}(22,6)$ que corresponde al último año de la educación básica primaria en Colombia. En los últimos tres grados académicos de la educación secundaria $\left(9^{\circ}=3,2 \% ; 10^{\circ}=12,9 \%\right.$; $11^{\circ}=0 \%$ ) no se identifica una tendencia ascendente o descendente en los resultados, aunque se observa que el grado $10^{\circ}$ presenta el mayor nivel $(12,9 \%)$ entre estos.

Para el caso del hostigamiento escolar realizado a través de medios virtuales, se evidencia que el número de víctimas en los dos grados escolares de educación básica tenidos en cuenta $\left(4^{\circ}=15,8 \%\right.$; $5^{\circ}=15,8 \%$ ) es la misma cantidad; en los grados $6^{\circ}$ y $7^{\circ}$ también se observa el mismo número de víctimas entre estos $(5,3 \%)$, ascendiendo notablemente en el grado $8^{\circ}(26,3)$; mientras en los últimos tres grados escolares $\quad\left(9^{\circ}=5,3 \% ; \quad 10^{\circ}=15,8 \% ; \quad 11^{\circ}=10,5 \%\right)$, $10^{\circ}$ es el que presenta mayor prevalencia. Los anteriores resultados hallados a un nivel descriptivo, supondrían la necesidad de brindar mayor interés de los programas de convivencia escolar en el grado $8^{\circ}$, para los dos tipos de hostigamiento escolar (personal y virtual), sin embargo, la prevención e intervención del hostigamiento escolar deben permear toda la institución educativa.

De otra parte, se destaca que el $9,2 \%$ de la muestra $(n=1776)$ refiere haber sentido miedo de asistir a la escuela, siendo esta cifra $1,1 \%$ mayor a la de los estudiantes que son agredidos de manera personal y 4,2\% mayor al de los estudiantes víctimas de hostigamiento a través de medios virtuales, lo que indica que en las instituciones públicas de Bucaramanga, más estudiantes de los que son agredidos, se encuentran intimidados ante el hostigamiento escolar, aunque no sean víctimas directas, tal como se ha evidenciado en otros estudios (Chaux, 2012; Wang, Iannotti, \& Nansel, 2009). 


\section{Conclusiones}

Para concluir, es necesario señalar que la cooperación entre los diferentes actores de la comunidad educativa es fundamental para el desarrollo de programas y políticas de prevención e intervención del hostigamiento escolar. La formación en valores y ciudadanía recibidas en el hogar, fortalece la capacidad de afrontamiento y las habilidades sociales de los estudiantes; los centros educativos deben preocuparse por generar una cultura organizacional propia, en la que se establezcan valores cívicos $\mathrm{y}$ morales que enmarquen las relaciones sociales que se den al interior de la institución; y el estado debe procurar una actitud proactiva de apoyo para la comunidad educativa de la que es parte (Santos, 2006).

Finalmente, es importante que los resultados de esta investigación deban ser interpretados con cautela debido a su naturaleza transversal: un estudio longitudinal fortalecería los planteamientos aquí hechos. Asimismo, teniendo en cuenta que el proceso de recolección de información se realizó mediante autorreporte, las respuestas podrían presentar sesgos (García \& Gracia, 2009); sin embargo, esta investigación resulta un aporte considerable sobre el conocimiento del hostigamiento escolar y destaca la importancia de continuar estudiando el fenómeno, considerando aspectos adicionales como el envolvimiento de los padres y las características de las instituciones educativas, pues las acciones de prevención del hostigamiento escolar deben considerar el conocimiento formal de toda la comunidad educativa sobre el fenómeno (Moura, Cruz, \& Quevedo, 2011).

\section{Agradecimientos}

La presente investigación está enmarcada en el desarrollo del proyecto "Efectividad de los programas OBPP - Bergen y Save en la disminución del hostigamiento escolar en instituciones públicas de educación básica de Bucaramanga" financiado por el Departamento Administrativo de Ciencia Tecnología e Innovación Colciencias y la Universidad Industrial de Santander y es avalada por la Vicerrectoría de Investigación y Extensión de dicha institución bajo el código 8246.
Esta investigación fue posible gracias a la participación activa los directivos, docentes, padres/ madres y estudiantes de las instituciones educativas públicas del municipio de Bucaramanga.

\section{Referencias bibliográficas}

Amemiya, I; Oliveros, M. \& Barrientos, A. (2009). Factores de riesgo de violencia escolar (bullying) severa en colegios privados de tres zonas de la sierra del Perú. Anales de la Facultad de Medicina, 70(4), 255-8.

Aguirre, F. (2009). Violencia Escolar y Política Educativa en el Perú. Lima: Universidad Alberto Hurtado-Cide.

Avilés, J. (2009a). Victimización Percibida y Bullying - Factores Diferenciales entre Víctimas. Boletín de Psicología, 95 (1), 7-28.

- (2009b). Ciberbullying Diferencias entre el alumnado de secundaria. Boletín de Psicología, 96, 79-96.

Avilés, J. M. \& Monjas, I. (2005). Estudio de la incidencia de la intimidación y el maltrato entre iguales en la educación secundaria obligatoria mediante el cuestionario CIMEI (Avilés, 1999) - Cuestionario Sobre Intimidación y Maltrato Entre iguales. Anales de Psicología, $21(1), 27-41$.

Batsche, G. \& Knoff, H. (1994). Bullies and their victims: understanding a pervasive problem in the schools. School Psychology Review, 23, $165-175$.

Buelga, S., Cava, M. \& Musitu, G. (2010). Cyberbullying: victimización entre adolescentes. Psicothema, 22 (4), 784-789.

Cepeda-Cuervo, E., Pacheco-Durán, P., García-Barco, L. \& Piraquive-Peña, C. (2008). Acoso Escolar a Estudiantes de Educación Básica y Media. Revista Salud Pública, 10 (4), $517-528$.

Chagas, R. (2005). Los maestros frente a la violencia entre alumnos. Revista Mexicana de Investigación Educativa, 10 (27), 1071 1082. 
Chaux, E. (2012). Educación, convivencia y agresión escolar. Bogotá: Taurus - Universidad de Los Andes.

Debardieux, E \& Blaya, C. (2001). La violence en milieu scolaire. Dix aproches en Europe. París: ESF.

Defensor del Pueblo (1999). Informe del Defensor del Pueblo Sobre Violencia Escolar. Madrid, España.

Díaz-Aguado, M. (2005). La violencia entre iguales en la adolescencia y su prevención desde la escuela. Psicothema, 17 (4), 549-558.

Gaviria, S. et al. 2012. Proyecto de ley por la cual se crea el sistema nacional de convivencia escolar y formación para el ejercicio de los derechos humanos, sexuales y reproductivos y la prevención y mitigación de la violencia escolar. (Proyecto en curso). Bogotá.

García, F \& Gracia, E. (2009). Is always authoritative the optimum parenting style? Evidence from Spanish families. Adolescence, 44 (173), 101-131.

García, B \& Ortiz, B. (2012). Los maestros ante la violencia escolar. Bogotá: Universidad Distrital Francisco José de Caldas.

García, B Guerrero, J. y Ortiz, B. (2012). La violencia escolar en Bogotá desde la mirada de las familias. Bogotá: Universidad Distrital Francisco José de Caldas.

Instituto Nacional de Salud Pública (2006). Encuesta Nacional de Salud y Nutrición de 2006 México. Recuperado de http://ensanut. insp.mx/informes/ensanut2006.pdf

Kamen, C. et al. (2012). The impact of childhood bullying among HIV-positive men: psychosocial correlates and risk factors. Child abuse \& neglect, 37(4), 273-281.

Ley 1620 del 2013, Por la cual se crea el sistema nacional de convivencia escolar y formación para el ejercicio de los derechos humanos, la educación para la sexualidad y la prevención y mitigación de la violencia escolar.
Oliveros, M; Barrientos,A. (2007). Incidencia y Factores de riesgo de la Intimidación "Bullying" en un Colegio particular de Lima, Perú. Revista Peruana de Pediatría, 60 (3), 150-155.

Oliveros, M., Figueroa, L., Mayorga, G., Cano, B., Quispe, Y. \& Barrientos, A. (2008). Violencia escolar (bullying) en colegios estatales de primaria en el Perú. Revista Peruana de Pediatría, 61(4), 215 - 220.

Olweus, D. (1993) Bullying at school. Cambridge, MA, Blackwell Publishers, 273-285.

(1998). Conductas de acoso y amenaza entre escolares. Madrid: Ediciones Morata.

(2007). Acoso Escolar: Hechos y Medidas de Intervención. Research Centre forHealthPromotion. Universidad de Bergen, Noruega.

Ortega, R. \& Del Rey, R. (2001). Aciertos y desaciertos del proyecto Sevilia Anti-Violencia escolar (SAVE). Revista de Educación, 324, 253-270.

Ortega, R. (1998). Indiscipline or violence? The problem of bullying in school. Prospects, 28(4), 587-599.

Paredes, M; Álvarez, M., Lega, L. \& Vernon, A. (2008). Estudio exploratorio sobre el fenómeno del "Bullying" en la ciudad de Cali, Colombia. Revista Latinoamericana de Ciencias Sociales, Niñez y Juventud, 6 (1), 295-317.

Martín, P. Lobato, H. \& Gómez, A. (2007). La percepción ante el fenómeno "bullying" de los profesores en formación. Wanceulen Digital, 3.

Milicic, N. \& López, S. (2008). Hostigamiento escolar: propuestas para la elaboración de políticas públicas. Santiago de Chile: Pontificia Universidad Católica de Chile, Vicerrectoría de Comunicaciones y Asuntos Públicos. 
Moreno, J. (2007). Comportamiento Antisocial en los Centros Escolares: Una Visión desde Europa. Revista Iberoamericana de Educación, 18, 189-206.

Moura, D; Cruz, A. \& Quevedo, L. (2011). Prevalence and characteristics of school age bullying victims. Jornal de Pediatria, 87 (1), 19-23.

Pérez-Fuentes, M., Gázquez, J., Fernández-Baena, R. \& Molero, M. (2011). Análisis de las publicaciones sobre convivencia escolar en una muestra de revistas de educación en la última década. Aula Abierta, 39 (2), 81-90.

Recha, R., Halpernb, R., Tedescoc, A. \& Santosd, D. (2013). Prevalence and characteristics of victims and perpetrators of bullying. Jornal de Pediatria, 89 (2), 164-170.

Rodríguez, J.M. (2009). Acoso escolar - Medidas de Prevención y Actuación. Educacao, Porto Alegre. 32 (1), 51-58.

Romera, E., Del Rey, R. \& Ortega, R. (2011). Prevalencia y aspectos diferenciales relativos al género del fenómeno bullying en países pobres. Psicothema, 23 (4), 624-629.
Santos M.A. (2006) Currículum oculto y aprendizaje en valores. Universidad de Málaga. El Refugio 27.05.2006. disponible en URL: el-refugio.net/index. php?option $=$ com content\&task=view\&id=240\&Itemid

Serrano, A \& Iborra, I. (2005). Violencia entre compañeros en la escuela. Centro Reina Sofía para el estudio de la violencia. España. Goaprint, S.L.

Solberg, M \& Olweus, D. (2003). Prevalence estimation of school bullying with the Olweus Bylly/Victim Questionnaire. Aggressive Behavior, 29, 239 - 268.

Wang, J; Iannotti, R. \& Nansel, T. (2009). School bullying among adolescents in the United States: physical, verbal, relational, and cyber. Journal of Adolescent Health, 45(4), 368-375.

Uribe,A; Orcasita, L \& Aguillon, E. (2012). Bullying, social support networks and family functioning in adolescents in an educational institution Santander, Colombia. Psycología, Avances de la disciplina [online]. 6 (2), 83-99.

Whitney, I. \& Smith, P. K. (1993). A survey of the nature and extent of bullying in junior/ middle and secondary schools. Educational research. 35(1), 3-25. 